\title{
BCG vaccination in humans inhibits systemic inflammation in a sex-dependent manner
}

Valerie A.C.M. Koeken, ${ }^{1,2}$ L. Charlotte J. de Bree, ${ }^{1,2,3,4}$ Vera P. Mourits, ${ }^{1,2}$ Simone J.C.F.M. Moorlag, ${ }^{1,2}$ Jona Walk, ${ }^{1,2,5}$ Branko Cirovic, ${ }^{6}$ Rob J.W. Arts, ${ }^{1,2}$ Martin Jaeger, ${ }^{1,2}$ Helga Dijkstra, ${ }^{1,2}$ Heidi Lemmers, ${ }^{1,2}$ Leo A.B. Joosten,, ${ }^{1,2}$ Christine S. Benn,,$^{3,4}$ Reinout van Crevel, ${ }^{1,2}$ and Mihai G. Netea ${ }^{1,2,6}$

'Radboud Center for Infectious Diseases and 2Department of Internal Medicine, Radboud University Medical Center, Nijmegen, Netherlands. ${ }^{3}$ Bandim Health Project, OPEN, Institute of Clinical Research, University of Southern Denmark/Odense University Hospital, Odense, Denmark. ${ }^{4}$ Danish Institute for Advanced Study, University of Southern Denmark, Odense, Denmark. ${ }^{5}$ Department of Medical Microbiology, Radboud University Medical Center, Nijmegen, Netherlands. ${ }^{6}$ Quantitative Systems Biology, Life and Medical Sciences Institute (LIMES), University of Bonn, Bonn, Germany.

BACKCROUND. Induction of innate immune memory, also termed trained immunity, by the antituberculosis vaccine bacillus Calmette-Guérin (BCC) contributes to protection against heterologous infections. However, the overall impact of BCC vaccination on the inflammatory status of an individual is not known; while induction of trained immunity may suggest increased inflammation, BCG vaccination has been epidemiologically associated with a reduced incidence of inflammatory and allergic diseases.

METHODS. We investigated the impact of BCG (BCG-Bulgaria, InterVax) vaccination on systemic inflammation in a cohort of $\mathbf{3 0 3}$ healthy volunteers, as well as the effect of the inflammatory status on the response to vaccination. A targeted proteome platform was used to measure circulating inflammatory proteins before and after BCG vaccination, while ex vivo Mycobacterium tuberculosis- and Staphylococcus aureus-induced cytokine responses in peripheral blood mononuclear cells were used to assess trained immunity.

RESULTS. While BCG vaccination enhanced cytokine responses to restimulation, it reduced systemic inflammation. This effect was validated in 3 smaller cohorts, and was much stronger in men than in women. In addition, baseline circulating inflammatory markers were associated with ex vivo cytokine responses (trained immunity) after BCG vaccination.

CONCLUSION. The capacity of BCG to enhance microbial responsiveness while dampening systemic inflammation should be further explored for potential therapeutic applications.

FUNDING. Netherlands Organization for Scientific Research, European Research Council, and the Danish National Research Foundation.

\section{Introduction}

The traditional view of vaccines is that they protect against a particular infection by induction of long-lasting specific adaptive immune memory. The discovery of the induction of nonspecific innate immune memory (also termed trained immunity) by the antituberculosis vaccine bacillus Calmette-Guérin (BCG) has led to a paradigm shift in our understanding of our immune system $(1,2)$. BCG vaccination can induce epigenetic modifications and metabolic rewiring of monocytes, resulting in increased cytokine responses upon subsequent unrelated-pathogen chal-

\section{Related Commentary: p. 5121}

Authorship note: VACMK and LCJDB contributed equally to this work Conflict of interest: MGN and LABJ are scientific founders of Trained Therapeutics Discovery.

Copyright: (5) 2020, American Society for Clinical Investigation.

Submitted: September 30, 2019; Accepted: July 14, 2020;

Published: September 21, 2020.

Reference information: J Clin Invest. 2020;130(10):5591-5602.

https://doi.org/10.1172/JCl133935. lenge, up to 1 year after vaccination (3-6). The longevity of this effect is explained by BCG-induced reshaping of hematopoietic stem and progenitor cells within the bone marrow compartment, resulting in long-lasting transcriptional changes associated with myeloid cell development and function $(3,4)$. Recently, BCG vaccination was shown to reduce Plasmodium falciparum parasitemia in a controlled human malaria infection model (5), as well as yellow fever viremia following yellow fever vaccination (6), suggesting that BCG-induced trained immunity also contributes to enhanced overall protection of infants after BCG vaccination (7-12). However, future studies are warranted to assess the duration of these effects.

Although studies so far suggest that BCG vaccination elicits enhanced activation only upon subsequent reinfection $(5,6)$, concerns may be raised that BCG could promote a proinflammatory environment facilitating the development of inflammationmediated diseases such as atherosclerosis (13-15) and autoimmune and autoinflammatory diseases $(16,17)$. On the other hand, epidemiological studies suggest that BCG vaccination reduces the risk of atopy, eczema, and asthma (18-22), diseases in which 


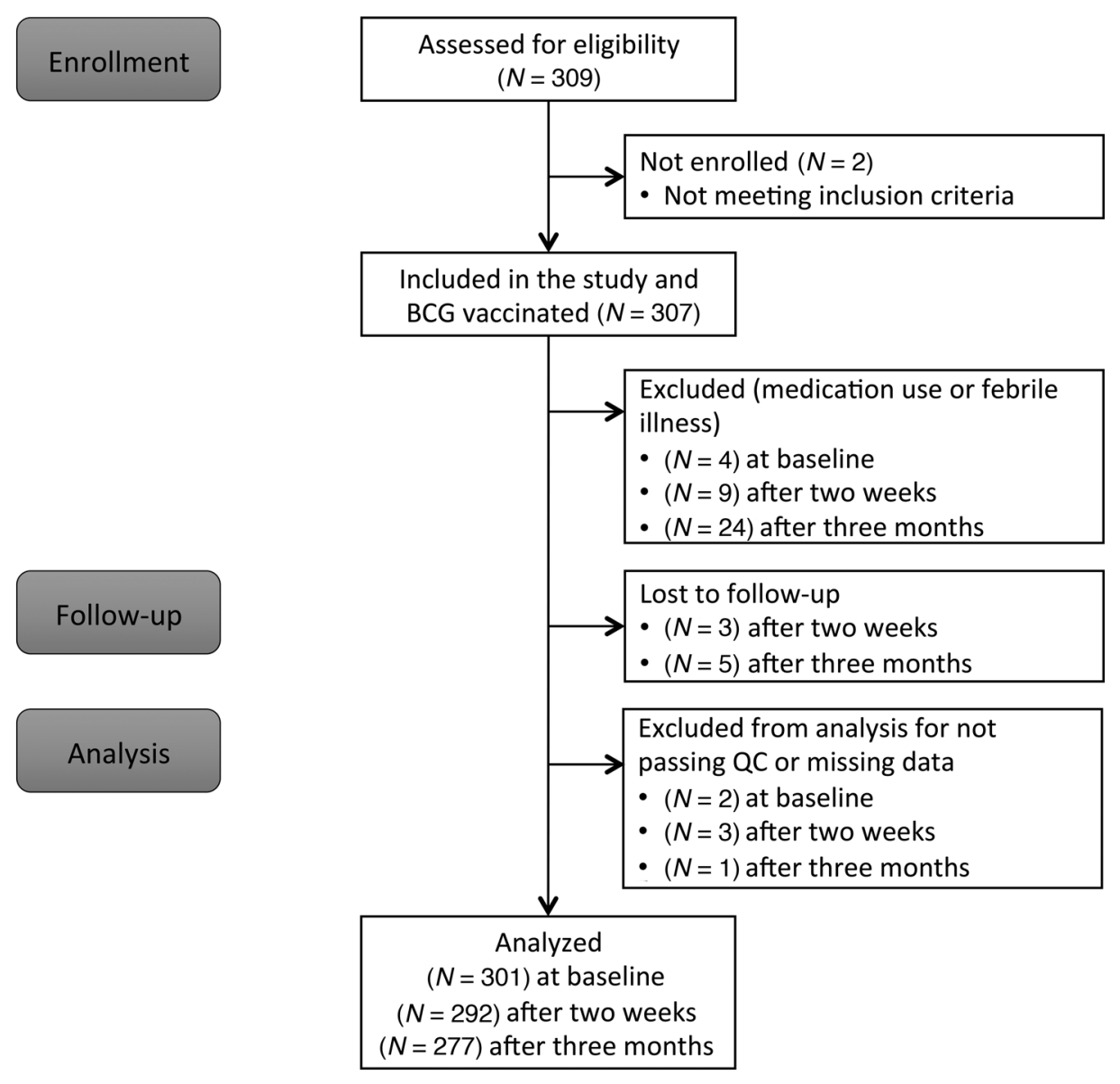

Figure 1. Flow chart of the study. Flow diagram describing the number of participants who were enrolled in the study, who were excluded or dropped out of the study, or excluded from further analysis.

inflammation plays an important role. Therefore, the impact of BCG vaccination on the inflammatory status of an individual needs to be explored. In addition, BCG-induced specific $(23,24)$ and nonspecific $(7,25)$ protective effects vary across different settings and are variable between healthy volunteers $(5,6,26)$. Understanding this variability may help identify individuals that will specifically benefit from BCG vaccination, or other interventions aimed at induction of trained immunity $(2,27)$. The prevaccination inflammatory status may contribute toward observed variability in immune responses after BCG vaccination, as was recently shown for hepatitis $\mathrm{B}$ (28) and yellow fever vaccination (29). Therefore, we aimed to investigate the interaction between inflammation and BCG vaccination by assessing a comprehensive set of circulating inflammatory biomarkers before and after BCG vaccination in a cohort of 303 healthy volunteers from the Human Functional Genomics Project (300-BCG cohort, www. humanfunctionalgenomics.org), and we validated the findings in 3 independent cohorts.

\section{Results}

$B C G$ vaccination downregulates circulating inflammatory markers. A total of 307 healthy volunteers were included in the 300BCG cohort. Four participants were excluded from further analysis due to medication use, resulting in 303 volunteers who completed the first visit (Figure 1). Fifty-six percent of the participants were female, the mean age was 26 years (range 18-71), and the mean body mass index (BMI) was 22.5 ( $\pm 2.6 \mathrm{SD}) \mathrm{kg} / \mathrm{m}^{2}$. BCG scars (data available for 286 volunteers) developed in 271 individuals (95\%), with a mean size of $0.42 \mathrm{~cm}( \pm 0.17 \mathrm{SD}) 3$ months after vaccination.

A targeted proteome platform was used to measure 92 inflammatory markers before $(n=301)$, and 2 weeks $(n=292)$ and 3 months $(n=277)$ after BCG vaccination. The quality of the measurement was high, with $99 \%$ of the samples passing quality control. Overall, 73 of the 92 (79\%) proteins were detected in at least $75 \%$ of the plasma samples and included in the analysis. Numerous baseline circulating inflammatory proteins showed a positive correlation with baseline whole blood counts of immune cell subsets (Figure 2A), as exemplified by the association of circulating oncostatin $\mathrm{M}$ (OSM) concentrations and neutrophil counts (Figure 2B) or circulating IL-6 concentrations and monocyte counts (Figure 2C). The fact that a number of inflammatory mediators are associated with cell counts is not necessarily surprising, as a significant number of the inflammatory mediators are correlated with each other, and in turn inflammation is associated with immune cell numbers in the blood. Surprisingly however, one-third of the proteins (25 out of 73) showed a significant decrease 2 weeks after BCG vaccination (Figure $3 \mathrm{~A}$ ). Of the 25 proteins with significantly reduced concentrations at 2 weeks, 10 remained lower 3 months after BCG vaccination (Figure $3 \mathrm{~B}$ ), such as TNF ligand superfamily member 12 (TWEAK) and sirtuin 2 (SIRT2) (Figure 3, C and D). No circulating protein was found to be significantly increased 3 months after BCG vaccination. An overview of fold changes in circulating inflammatory proteins is included in Supplemental 

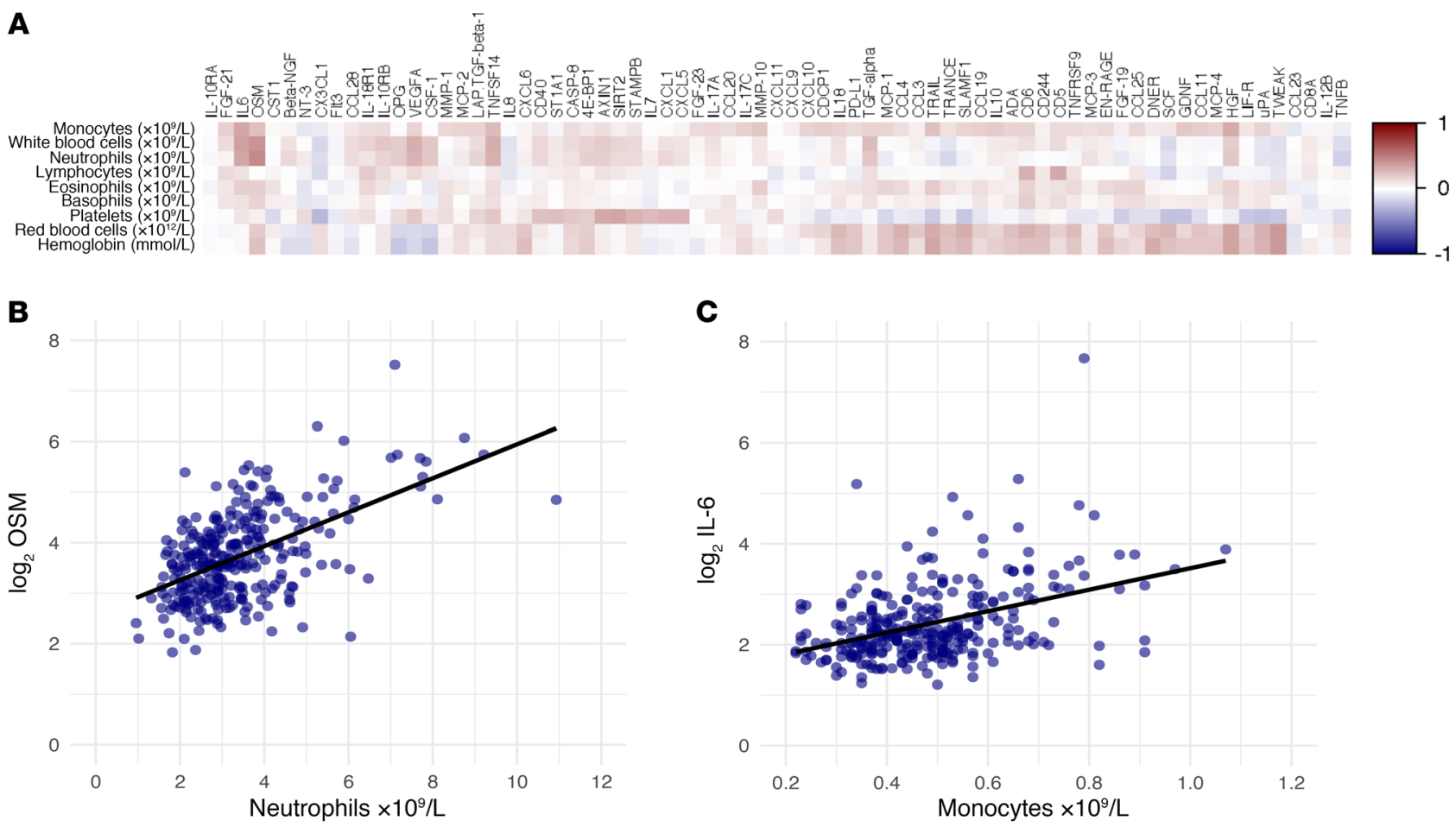

Figure 2. Correlations between baseline inflammatory markers and baseline whole blood counts. (A) Spearman's correlations between absolute whole blood counts (monocytes, total white blood cells, neutrophils, lymphocytes, eosinophils, basophils, platelets, red blood cells, and hemoglobin) and circulating inflammatory markers at baseline (before BCG vaccination). Positive correlations are depicted in red, negative correlations in blue ( $n=302)$. Spearman's correlations between whole blood neutrophil counts and circulating oncostatin M (OSM) (B), and between whole blood monocyte counts and circulating IL-6 (C) are shown as examples of positive correlations $(n=300)$.

Table 1 (supplemental material available online with this article; https://doi.org/10.1172/JCI133935DS1).

As an internal validation for a true effect of BCG vaccination, fold changes in significantly changed proteins were compared between BCG scar-positive volunteers $(n=271)$ versus volunteers who did not develop a scar $(n=15)$. In total, 5 proteins differed significantly between scar-positive and scarnegative volunteers (Figure 4A), with all proteins being lower in individuals that had a scar 3 months after vaccination. SIRT2 is given as an example of a protein that is significantly lower in scar-positive individuals 3 months after BCG vaccination in Figure 4B. Only a small proportion of participants (5\%) did not develop a scar. Therefore, the circulatory proteins that significantly changed after BCG vaccination were also correlated to scar size. In addition to the 5 proteins that showed a significant difference between scar-negative and scar-positive volunteers, 5 other proteins (ADA, MCP-2, caspase-8, CCL23, and OPG) were also significantly associated with scar size. The direction of the effect was similar for all 10 proteins; an increase in scar size was associated with a stronger reduction in the circulatory protein after BCG vaccination, further supporting the finding that BCG-induced immunological effects result in decreased systemic inflammation.

To validate our findings, this identical set of proteins was determined before and after BCG vaccination in plasma samples of 39 adult volunteers from 3 independent BCG vaccination trials (4-6). Even within these small cohorts, we were able to validate our findings regarding reduced concentrations of ADA, TWEAK, delta- and notch-like epidermal growth factor-related receptor (DNER), and neurotrophin-3 (NT-3), and increased concentration of IL-17C after BCG vaccination (Figure 5).

$B C G$ vaccination affects whole blood cell counts. Considering the observed differences in inflammatory status after BCG vaccination, we evaluated whole blood counts before and after BCG vaccination. Although total white blood counts remained stable after vaccination, BCG vaccination induced a slight increase in both lymphocyte (median $1.87 \times 10^{9} / \mathrm{L}$ at baseline and $1.97 \times 10^{9} / \mathrm{L}$ at 2 weeks, $P=0.009$ ) and monocyte (median $0.47 \times 10^{9} / \mathrm{L}$ at baseline and $0.48 \times 10^{9} / \mathrm{L}$ at 2 weeks, $P=0.003$ ) counts, which returned to baseline between 2 weeks and 3 months after vaccination. In addition, red blood cell counts and hemoglobin levels showed a slight reduction 2 weeks after vaccination $(P<0.0001$ for both), eosinophil counts showed a slight increase up to 3 months $(P=0.002)$, and platelet counts a mild reduction 3 months after vaccination $(P=0.0005)$. An overview of median whole blood cell counts per visit and $P$ values is included in Supplemental Table 2.

Antiinflammatory effect of $B C G$ vaccination is sex dependent. We next examined the effect of age, sex, and cytomegalovirus (CMV) serostatus on the concentrations of inflammatory proteins. The median age of our study population was 23 years (range 18-71), and $80 \%$ of the cohort was 25 or younger. Of the 73 proteins analyzed in our cohort, 6 had a negative correlation with age, and 19 a positive correlation with age $(\mathrm{FDR}<0.05)$. We also measured 
A

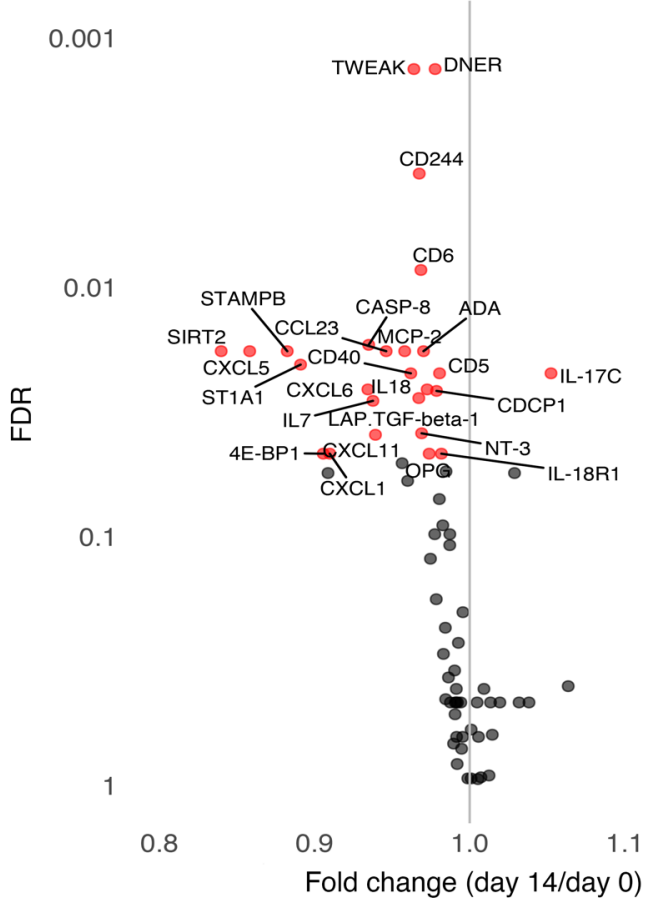

B

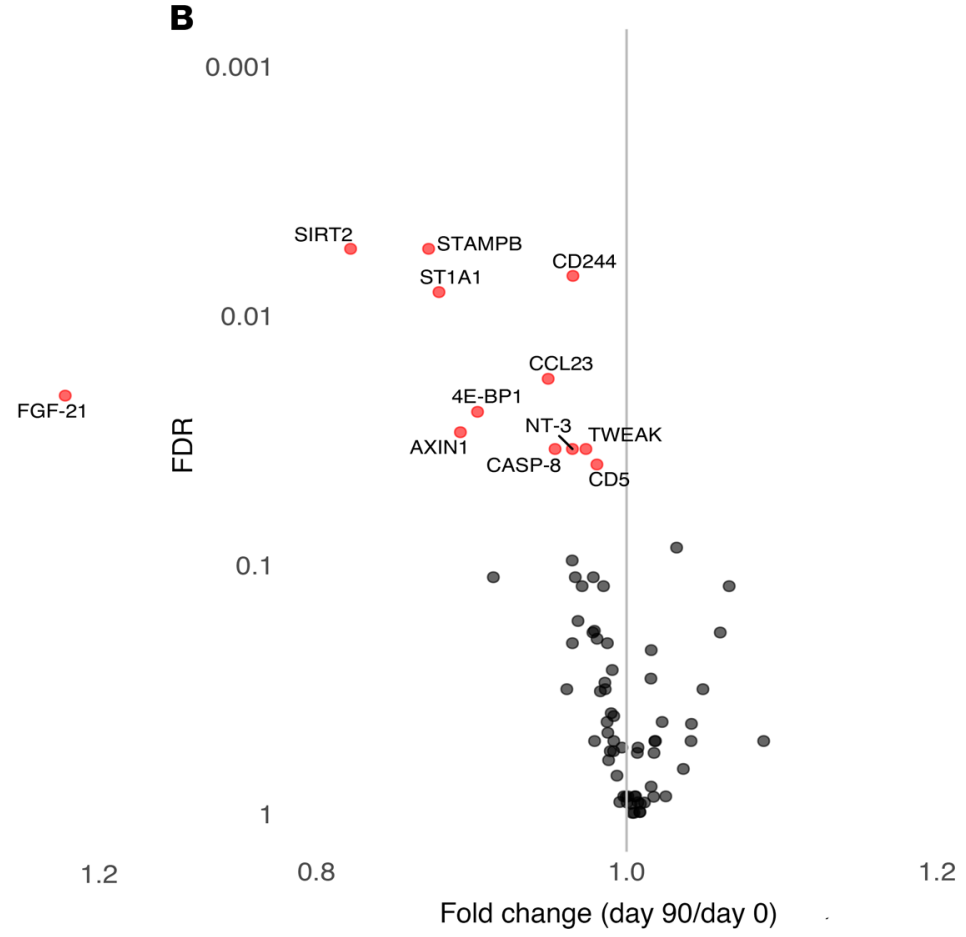

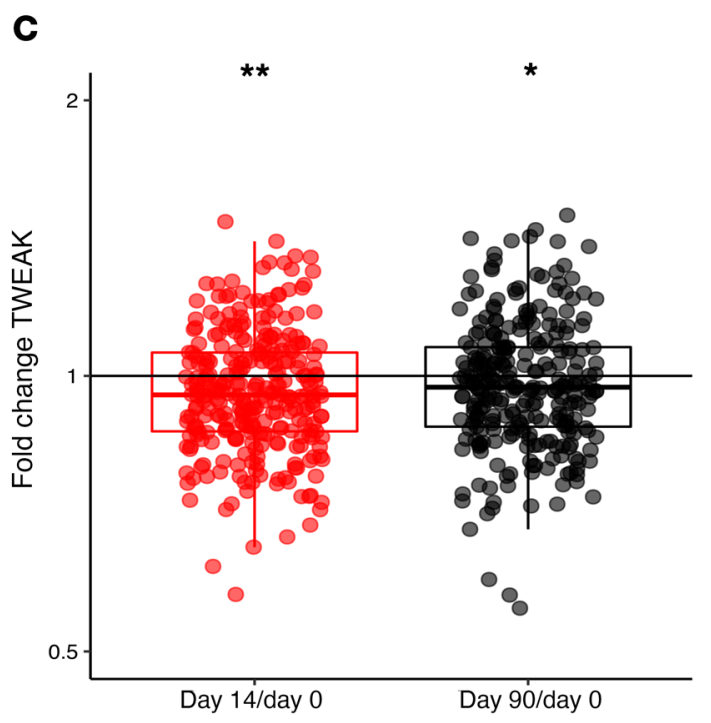

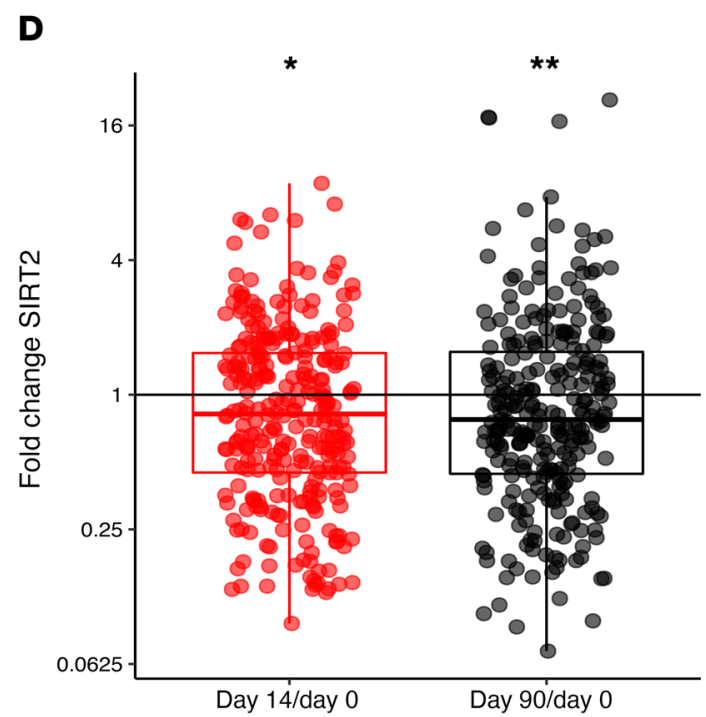

Figure 3. Inflammation after BCG vaccination. Fold changes in circulating inflammatory markers on day 14 versus baseline (A) and day 90 versus baseline (B). Significant changes compared with baseline are depicted in red, nonsignificant changes are depicted in gray ( $n=290$-fold change on day 14 versus baseline, $n=275$-fold change on day 90 versus baseline; FDR $<0.05$ is considered significant). Fold changes in TWEAK (C) and SIRT2 (D) are depicted as examples of significantly decreased circulating inflammatory markers after BCC vaccination ( ${ }^{*}$ FDR $<0.05,{ }^{* *}$ FDR $<0.01$ ).

CMV IgG in the plasma of our study participants. Five (CXCL9, FGF-19, TRAIL, CXCL10, and CXCL11) out of the 73 proteins analyzed in our study were higher in the CMV IgG-positive individuals $(24 \%)$ compared with the CMV IgG-negative individuals $(P<$ $0.05)$, but none of these differences were statistically significant after correction for multiple testing.

Stratified by sex (males $n=132$, females $n=171$ ), concentrations of inflammatory markers were significantly different between males and females at baseline (Figure 6A). Prevaccination concentrations of 41 inflammatory markers were significantly higher in males compared with females, and 6 proteins were found to be significantly lower in males compared with females. These sex-dependentdifferencesininflammatorymarkerswerevalidated in a second set of healthy volunteers (males $n=215$, females $n=$ 278). In total, 34 proteins were significantly different between males and females in both healthy cohorts (Figure 6B). Of these 34 , only 3 proteins were higher in females, while 31 were significantly higher in males.

Strikingly, the effect of BCG on systemic inflammation appeared to be much stronger in males, with 25 proteins showing 
A

\begin{tabular}{ll|l|l} 
A & & \multicolumn{2}{c}{ Scar } \\
& Fold change & No & Yes \\
\hline 4E-BP1 & Day 90 : Day 0 & 1.45 & 0.89 \\
\hline SIRT2 & Day 90 : Day 0 & 1.31 & 0.76 \\
\hline STAMPB & Day 90 : Day 0 & 1.20 & 0.83 \\
\hline AXIN1 & Day 90 : Day 0 & 1.51 & 0.87 \\
ST1A1 & Day 90 : Day 0 & 1.25 & 0.84
\end{tabular}

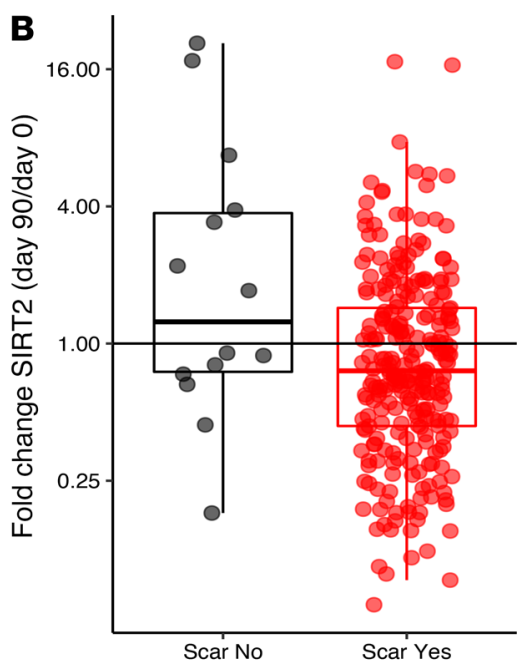

Figure 4. Differences in fold change in circulating proteins between scar-positive and scar-negative individuals. (A) Significant differences in fold changes in circulating proteins (significantly different in the entire cohort after FDR $<0.05$ correction) between scar-positive ( $n=290)$ and scar-negative $(n=15)$ individuals 90 days after vaccination. Fold changes higher than 1 depicted on a red scale, fold changes lower than 1 depicted on a blue scale. $P<$ 0.05 by Wilcoxon's matched-pairs signed-rank test. (B) SIRT2 was plotted as an example of a protein that is significantly lower in scar-positive individuals 3 months after BCG vaccination.

a significantly lower concentration 2 weeks after BCG vaccination after correction for multiple testing and none a higher concentration (Figure 6C). Three months after BCG vaccination, 16 of the 25 downregulated proteins at the 2-week time point remained significantly lower compared with baseline in males (Figure 6D). In contrast, no significant changes were found at 2 weeks or 3 months after BCG vaccination in females after correction for multiple testing. An overview of fold changes in circulating inflammatory proteins can be found in Supplemental Table 1.

Baseline characteristics such as age and BMI did not differ between males and females, but there were minor significant differences in red blood cell, platelet, monocyte, and eosinophil counts (Supplemental Table 3). In order to try to explain the sex-differential effects, we correlated circulating concentrations of adipokines (adiponectin, resistin, and leptin; higher in females) and testosterone levels (higher in males) with concentrations of the inflammatory proteins that were downregulated after BCG vaccination in males but not in females. Interestingly, baseline plasma testosterone showed a negative association with fold increase in several circulating inflammatory proteins after BCG vaccination in males (Figure 7A). This relationship is given as an example for CXCL1 in Figure 7B.

Correlation between inflammatory proteins and ex vivo cytokine production is sex dependent. We next examined if inflammatory protein profiles predicted ex vivo PBMC-derived cytokine production before and after BCG vaccination. Two weeks and 3 months after BCG vaccination, both Mycobacterium tuberculosis- and Staphylococcus aureus-induced production of innate cytokines was upregulated; S. aureus-induced TNF- $\alpha$ is given as an example in Figure $8 \mathrm{~A}$, in both men and women (sex-specific data not shown). Specific adaptive immune memory responses, as assessed by specific stimulation of IFN- $\gamma$ production with $M$. tuberculosis, were also upregulated by BCG vaccination (Figure 8B). At 2 weeks after vaccination, the increase in IFN- $\gamma$ in response to M. tuberculosis was significantly higher in females compared with males $(P=0.025$, Figure $8 \mathrm{C}$ ). No changes in lymphocyte or monocyte percentages within the PBMC fraction could be observed after BCG vaccination (Supplemental Table 4). Numerous prevaccination inflammatory proteins correlated with the increase in innate cytokine production capacity following BCG vaccination (Figure 8D). Circulating inflammatory proteins predominantly correlated with trained immunity responses 2 weeks after vaccination, and less with longer-term responses 3 months after vaccination. Interestingly, females mostly showed positive correlations whereas males only showed negative correlations between baseline circulating proteins and trained immunity responses following BCG vaccination, as for instance clearly shown for the relationships between plasma ADA, CD5, CD8a, IL-12B, TNFRSF9, and increase in S. aureusinduced IL-6. Especially in males, lower baseline concentrations of several inflammatory proteins were associated with increased M. tuberculosis-induced IL-1 $\beta$, IL- 6 , and TNF- $\alpha$ responses after BCG vaccination. In contrast, higher baseline concentrations of several other inflammatory proteins were associated with enhanced M. tuberculosis-induced IFN- $\gamma$ responses. Interestingly, circulating IL-10 showed a strong positive correlation with increased M. tuberculosis-induced IFN- $\gamma$ production after BCG vaccination in both males and females.

\section{Discussion}

BCG vaccination has important nonspecific effects by protecting against heterologous infections (7-12), being effective against bladder cancer (30), and reducing the risk of developing allergic and autoimmune diseases (18-22). However, while earlier studies have reported an upregulation of cytokine responses to nonspecific ex vivo restimulation (trained immunity), the impact of BCG vaccination on steady-state levels of inflammation was largely unknown. In this large cohort of healthy volunteers, we show that BCG vaccination enhances the capability of innate immune cells 
IL-17C

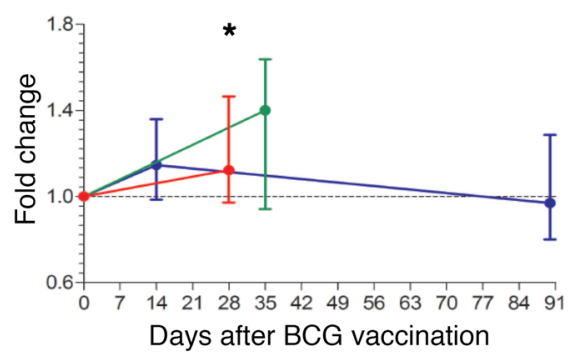

ADA

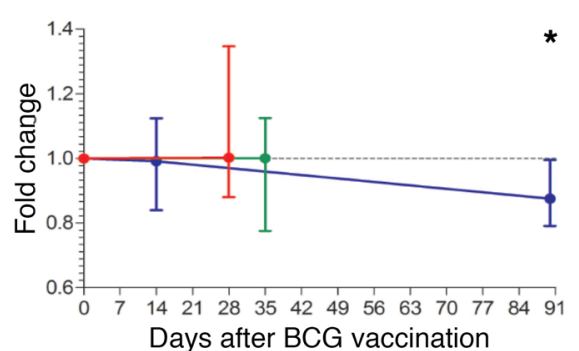

TWEAK

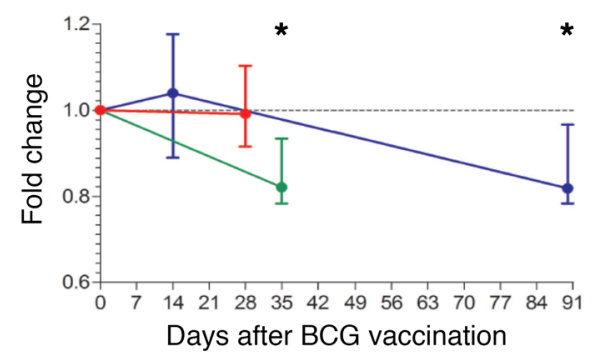

NT-3

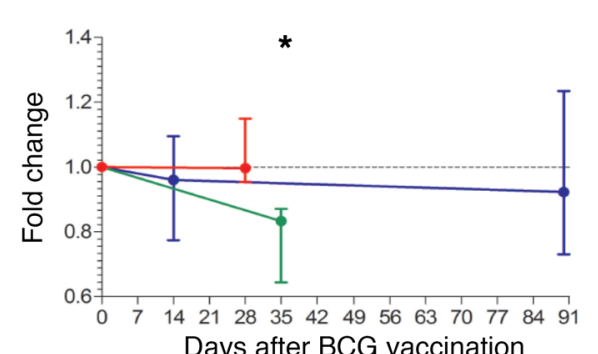

Days after BCG vaccination

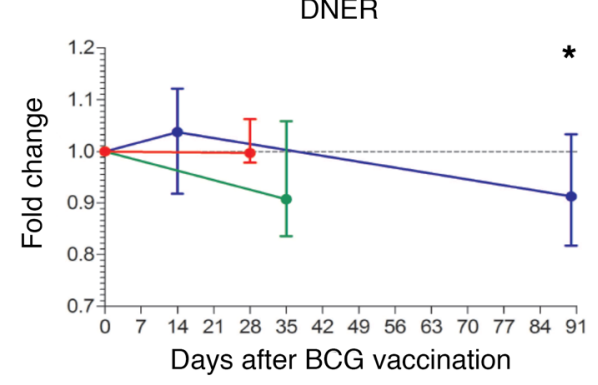

Days after BCG vaccination

Figure 5. Validation of changes in circulating proteins after BCG vaccination. Fold changes in circulating inflammatory markers (IL-17C, TWEAK, DNER, ADA, and NT-3) after BCG vaccination compared with baseline, validated in at least 1 of the 3 validation cohorts. The blue line represents cohort $1(n=15)$, the green line cohort $2(n=9)$, and the red line cohort $3(n=15) .{ }^{*} P<0.05$ by Wilcoxon's matched-pairs signed-rank test. Median \pm range is depicted per time point.

to respond with an antimicrobial response (assessed by cytokine production capacity), but at the same time downregulates the systemic inflammation as measured by decreased concentrations of proinflammatory proteins in the circulation of healthy volunteers.

This modulatory effect on systemic inflammation may explain some of the beneficial effects of BCG vaccination in inflammatory diseases. Induction of trained immunity by endogenous stimuli is believed to contribute to the development of atherosclerosis $(13-15,31)$, autoimmune and autoinflammatory diseases $(16,17)$, and due to the induction of trained immunity by BCG, one might hypothesize that BCG vaccination is a risk factor for these conditions. However, our results argue against a potentiating effect of BCG vaccination on inflammatory diseases such as atherosclerosis, and rather suggest that it may actually protect against inflammatory conditions. In support of this hypothesis, BCG vaccination in mice reduced the levels of circulating proinflammatory cytokines (32), lowered plasma cholesterol, and delayed the formation (33) and size of atherosclerotic lesions (32). Prospective studies have demonstrated beneficial effects of BCG vaccination in patients with autoimmune diseases such as multiple sclerosis (34-36) and type 1 diabetes mellitus $(37,38)$. Other studies have shown that BCG can prevent the development of diabetes in mice $(39,40)$. The reduction in circulating inflammatory markers following BCG vaccination might also contribute to the lower reported incidence of atopy and allergy after BCG vaccination $(18,19)$. Although a large prospective BCG vaccination trial in Danish newborns showed no effect on the incidence of atopy at the age of 13 months (41), a protective effect against atopic dermatitis was observed (22), in line with previous findings from a Dutch trial (21). Finally, a recent report suggested a beneficial effect of BCG on the devel- opment of Alzheimer's disease (42) in which downregulating inflammatory processes might play a role (43).

It remains unknown how BCG reduces overall inflammation while at the same time improving myeloid, NK, and heterologous $T$ cell responsiveness to microbial challenges. Earlier studies have focused on the gene sets that are upregulated during BCG vaccination in human myeloid cells. It is imperative that future studies extend these investigations in 2 directions: to evaluate the genes sets that are eventually silenced by BCG vaccination on the one hand, and to investigate the effects of BCG vaccination on nonimmune cells, which could also contribute to the release of inflammatory mediators on the other hand. In earlier studies from our group we have shown that, after induction of trained immunity, markers of both M1 and M2 macrophages are upregulated; we therefore proposed that the trained immunity phenotype is distinct from the classical M1/M2 dichotomy (44). In addition, earlier studies have shown that BCG vaccination tips the balance toward a more Th1-type response (45). A comprehensive assessment of these processes in follow-up studies is warranted.

Interestingly, we found a strong sex-differential effect, with significant reductions in inflammatory proteins after BCG vaccination in males only. Several studies have shown that both protective as well as detrimental nonspecific effects of vaccines are sex dependent $(10,46-51)$. Most of these consisted of observational studies, but 3 randomized trials have shown a sex-specific effect in all-cause morbidity and mortality after neonatal BCG vaccination (10). Within the first week after vaccination, strong protective effects were detected in boys, which waned after the first week, whereas in girls, the protective effect was limited in the first week, but more pronounced after the first week following vaccination (10). We found several negative correlations 
A

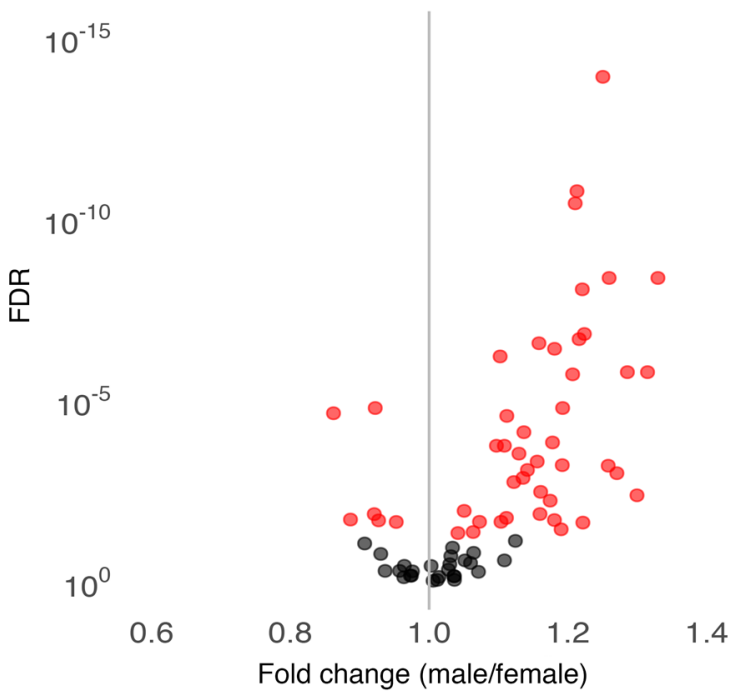

Fold change (male/female)

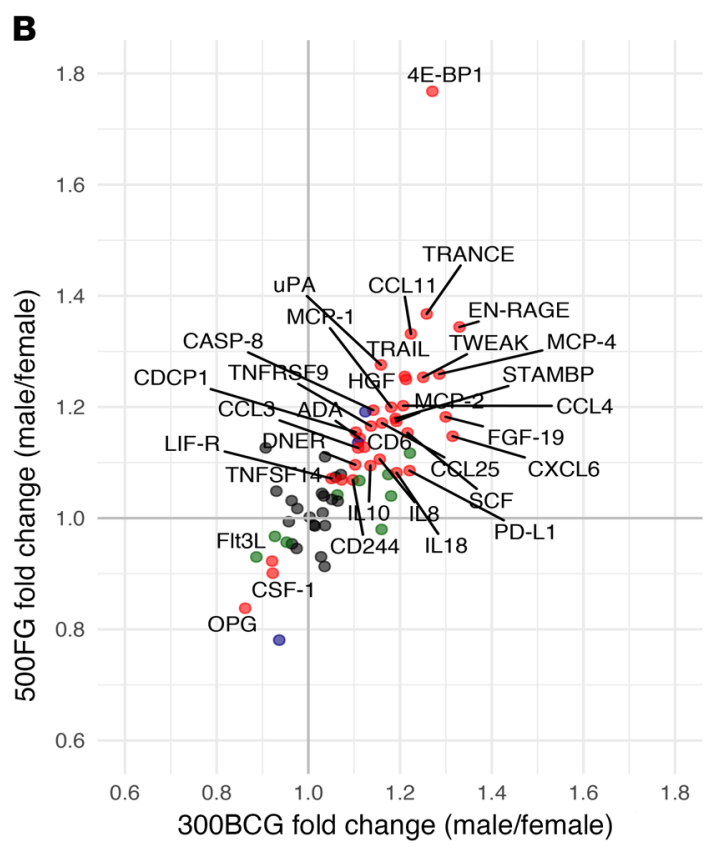

Significance

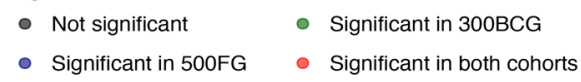

D

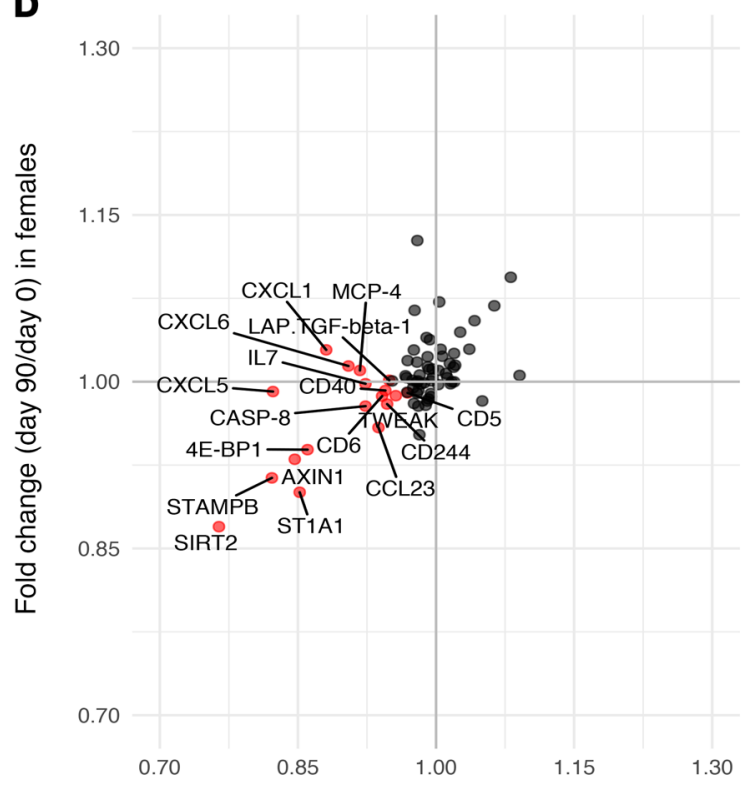

Fold change (day 90/day 0) in males

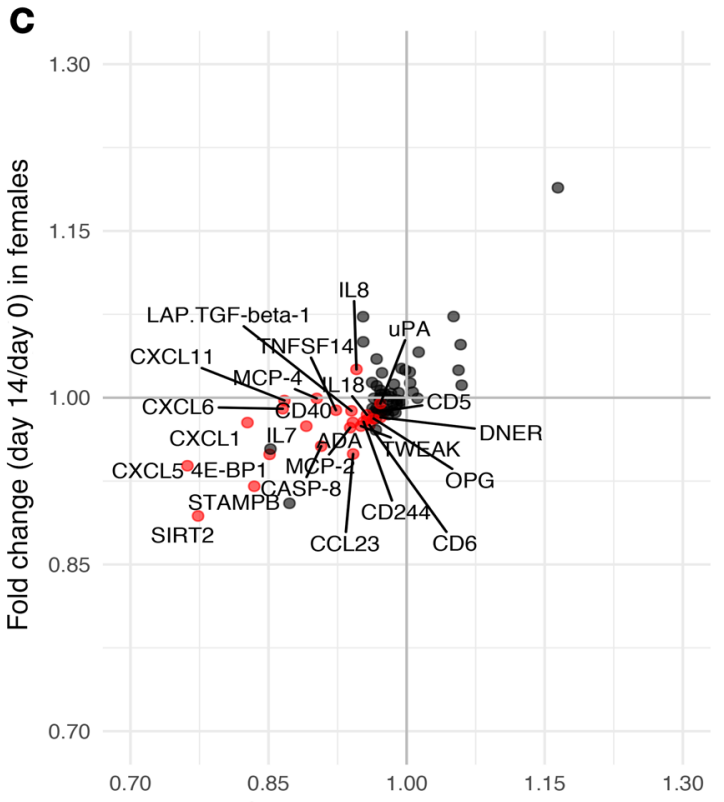

Fold change (day $14 /$ day 0 ) in males

Significance

- Not significant

- Only significant in males

Figure 6. Sex-specific effect of BCG vaccination on systemic inflammation. (A) Comparison of baseline circulating inflammatory proteins plotted as fold changes between males $(n=132)$ and females $(n=171)$. Significant changes between sexes are depicted in red (FDR $<0.05)$. (B) Comparison of inflammatory proteins between males and females from the discovery cohort (300BCG) were plotted against the comparison between males ( $n=215$ ) and females $(n=278)$ from the validation cohort (500FG). Proteins that were only significantly different in the 300BCG cohort are depicted in green ( $n=9$ ), those that were only significant in the 500FG cohort are depicted in blue $(n=3)$, and the proteins significantly different between males and females in both cohorts are depicted in red $(n=34)$ and are labeled with their name $(F D R<0.05)$. Fold changes of circulating inflammatory markers on day 14 versus baseline (C) and day 90 versus baseline (D) in the male-only $(n=132)$ versus the female-only $(n=171)$ subset. Significant changes compared with baseline in the male-only subset are depicted in red (FDR $<0.05)$, and proteins that did not significantly change after BCG vaccination in either the male-only or the female-only subset are depicted in gray. There were no proteins significantly different in the female-only subset. 
A

\begin{tabular}{llllll} 
A & Fold change & \multicolumn{2}{l}{ Testosterone Adiponectin } & Leptin & Resistin \\
4EBP1 & Day 14:Day 0 & $-0.20^{*}$ & -0.05 & 0.06 & -0.09 \\
CASP8 & Day 14:Day 0 & $-0.22^{*}$ & -0.1 & 0.04 & -0.02 \\
CD40 & Day 14:Day 0 & $-0.22^{*}$ & -0.03 & 0.00 & -0.05 \\
CD5 & Day 90:Day 0 & 0.08 & $0.19^{*}$ & 0.02 & 0.06 \\
CXCL1 & Day 14:Day 0 & $-0.29^{* * *}$ & -0.06 & 0.09 & 0.00 \\
CXCL5 & Day 14:Day 0 & $-0.26^{* *}$ & -0.05 & 0.03 & 0.00 \\
CXCL6 & Day 14:Day 0 & $-0.21^{*}$ & -0.02 & 0.06 & 0.03 \\
IL-7 & Day 14:Day 0 & $-0.22^{*}$ & -0.07 & 0.02 & 0.00 \\
OPG & Day 14:Day 0 & $0.22^{*}$ & -0.01 & -0.07 & 0.01 \\
SIRT2 & Day 14:Day 0 & $-0.27^{*}$ & -0.08 & 0.06 & -0.03 \\
ST1A1 & Day 90:Day 0 & $-0.21^{*}$ & 0.1 & -0.03 & -0.07 \\
STAMPB & Day 14:Day 0 & $-0.28^{* *}$ & -0.05 & 0.07 & -0.03
\end{tabular}

B

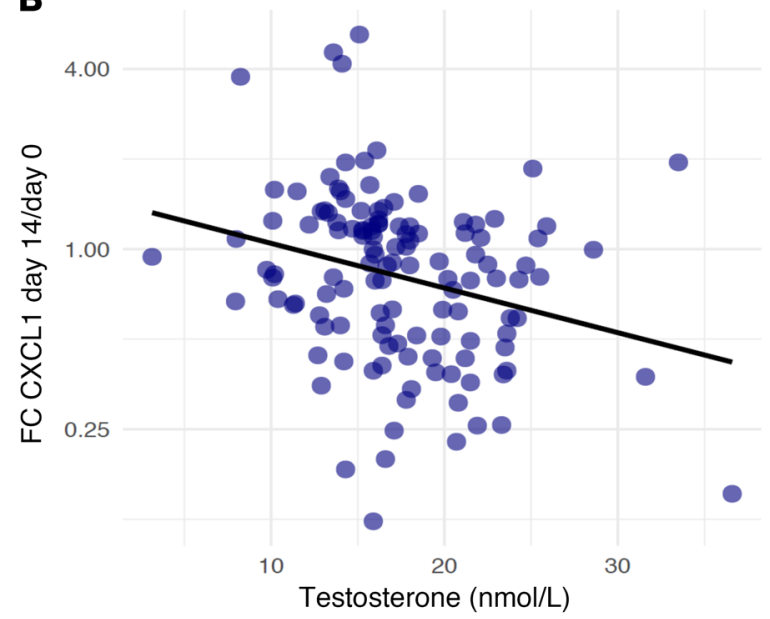

Figure 7. Correlations between circulating hormones and inflammatory proteins. (A) Fold changes of proteins that significantly changed after BCG vaccination in males were correlated to baseline testosterone, adiponectin, leptin, and resistin concentrations. Only proteins with a significant correlation with one of the hormones are depicted in this figure. ${ }^{*} P<0.05,{ }^{* *} P<0.01,{ }^{* *} P<0.001$ by Spearman's correlation. The color represents the strength and the direction of the correlation. (B) Spearman's correlation between testosterone at baseline and fold change in CXCL1 2 weeks after vaccination is shown as an example.

between baseline testosterone concentrations and changes in circulating proteins after BCG vaccination in males, suggesting a possible role for testosterone in our observed sex-differential effects after BCG vaccination. In line with these findings, previous in vitro studies have demonstrated that high dihydrotestosterone concentrations reduced monocyte-derived IL-6 and TNF- $\alpha$ production after 24 -hour stimulation with BCG (52). It remains to be investigated how our findings link to the observed sex differences in epidemiological studies, considering that BCG is mainly administered in neonates in tuberculosis-endemic countries, which is different from our study. It might seem evident that the mechanisms responsible for sex differences are different in young infants compared with adults due to the differences in sex hormones. Interestingly however, sex hormone levels peak shortly after birth, the so-called mini puberty (53), which is at the same time that neonates normally receive their BCG vaccination, indicating that sex hormones could modulate $\mathrm{BCG}$-induced effects in the neonatal period as well as later in life. Still, there might be different mechanisms operating in these different age groups, which is why future research should investigate these effects in other relevant populations.

Another important finding from this study is that the prevaccination inflammatory status alters both specific and nonspecific immune responses after BCG vaccination. Circulating inflammatory proteins mostly seem to potentiate short-term nonspecific effects of BCG vaccination (trained immunity responses 2 weeks after vaccination), and to a lesser extent the longer-term induction of trained immunity. Sex specificity was detected in the effect of baseline inflammation on induction of trained immunity. The lower the baseline inflammation in males, the better the training responses, whereas higher baseline inflammation in females was associated with an enhanced training phenotype. Considering the differences in concentrations of baseline inflammatory status between males and females, our results suggest that a certain optimum in prevaccination circulating inflammatory markers facilitates induction of trained immunity after BCG vaccination. In addition, baseline inflammation also impacts the longer-term effects of BCG vaccination on the induction of specific $M$. tuberculosis-induced cytokine responses. In males, we found a clear dichotomy in the associations between baseline inflammatory status and $M$. tuberculosis-induced cytokine responses after BCG vaccination: a predominantly negative association between several circulating inflammatory proteins and innate cytokine responses, and exclusively positive associations between circulating inflammatory markers and IFN- $\gamma$ responses. For specific adaptive responses, higher baseline concentrations of IL-10, IL-12B, and CXCL10 (also known as IFN- $\gamma$-induced protein 10), which has previously shown to be important in mycobacterial outgrowth and was identified as possible novel marker of trained immunity (54), resulted in increased $M$. tuberculosis-induced IFN- $\gamma$ responses. Our data are partially in line with previous observations that lower prevaccination inflammation enhances vaccine immunogenicity $(28,29)$, which in our case holds specifically true for induction of innate immune memory responses in males. Effects of prevaccination inflammatory status have recently been found for hepatitis B vaccination (28); a higher frequency of both activated innate immune cells and proinflammatory cytokines correlated with lower neutralizing antibody titers following HBV vaccination (28). Similarly, after yellow fever vaccination, baseline numbers of activated $\mathrm{CD} 8^{+} \mathrm{T}$ cells and $\mathrm{B}$ cells and proinflammatory monocytes resulted in lower neutralizing antibody titers following vaccination (29).

Our study is limited by the fact that we have used a focused proteomics platform. Future studies should expand these investigations by studying the effect of BCG vaccination at a broader level by untargeted proteomics. Moreover, longitudinal studies should focus on the risk of developing inflammatory diseases after BCG vaccination. 
A

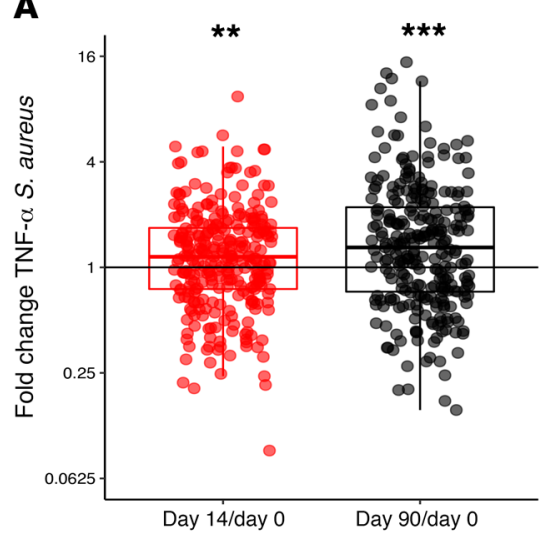

B

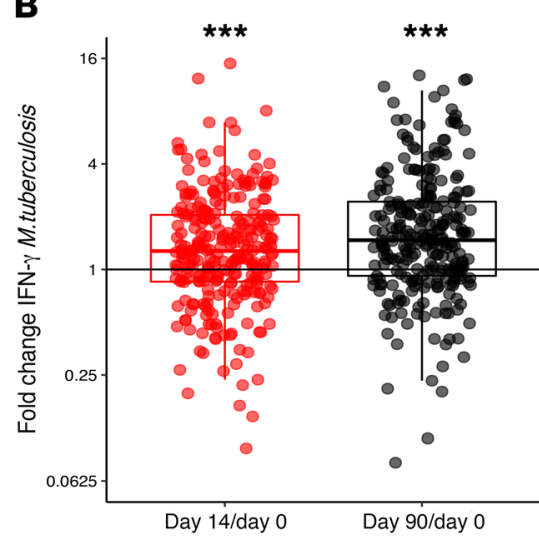

C

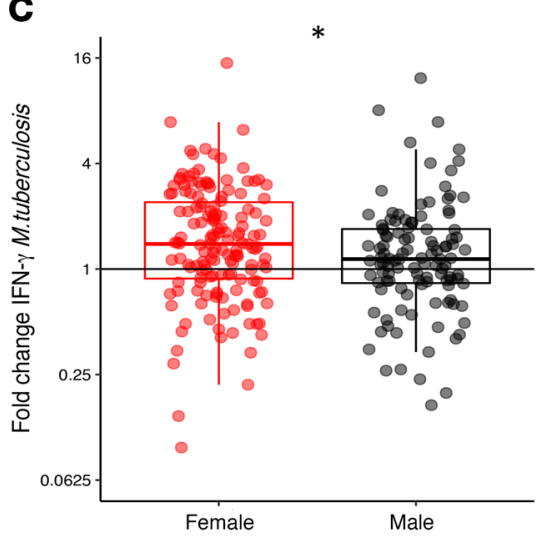

D

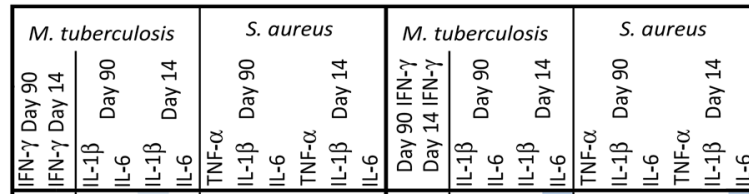

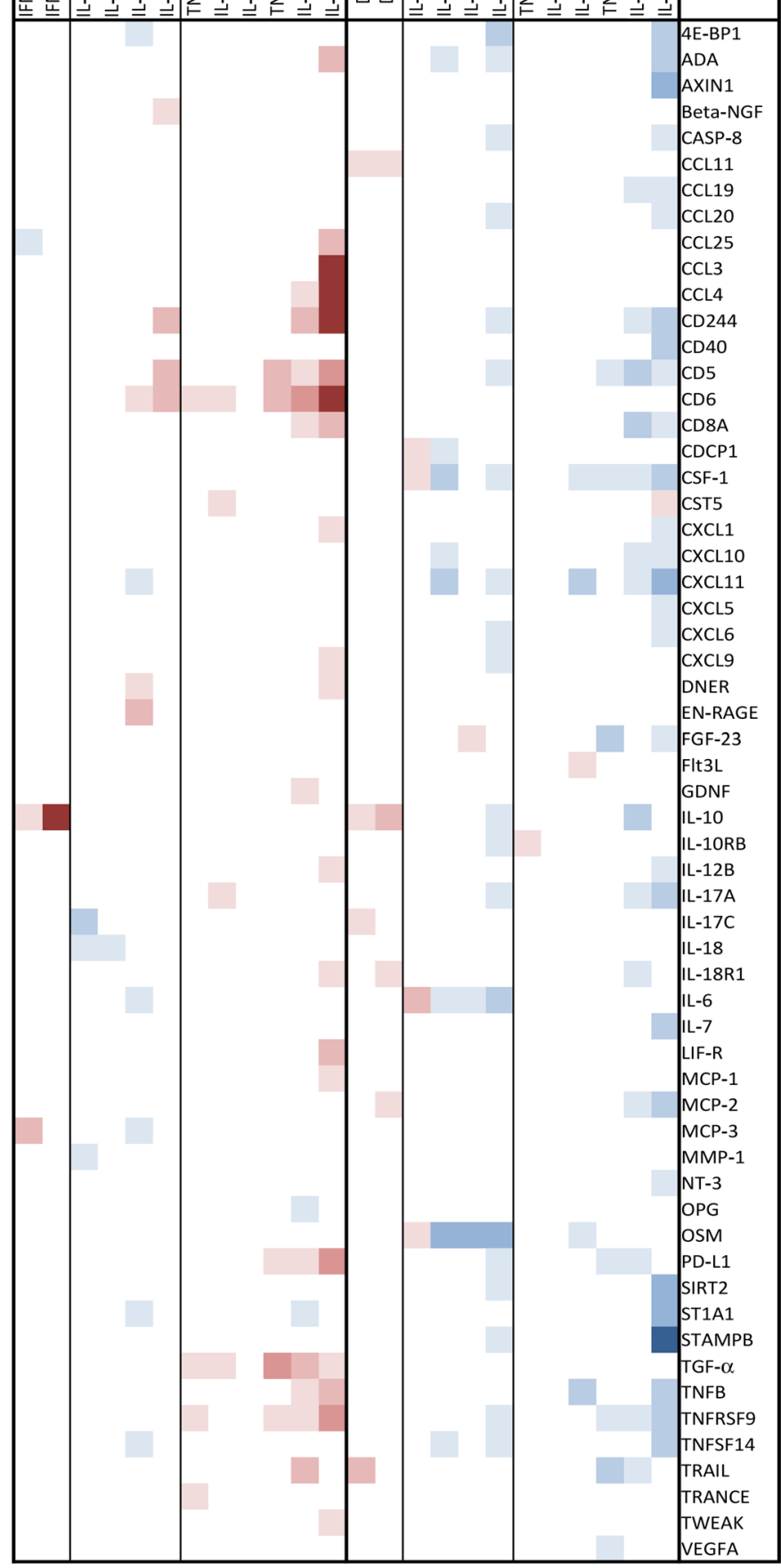

Female

Male

Figure 8. Ex vivo PBMC-derived cytokine production and associations with baseline circulating inflammatory proteins. Fold changes compared with baseline of ex vivo PBMC-derived S. aureus-induced TNF- $\alpha$ responses (A) and M. tuberculosis-induced IFN- $\gamma$ responses (B) on day 14 versus baseline and day 90 versus baseline as examples of upregulated cytokine responses after BCG vaccination (fold change day 14 versus baseline $n=289$, fold change day 90 versus baseline $n=275$ ). ${ }^{*} P<0.05$, ${ }^{*} P<0.01,{ }^{* *} P<0.001$ by Wilcoxon's matched-pairs signed-rank test. (C) Fold changes in IFN- $\gamma$ in response to M. tuberculosis on day 14 versus baseline separated by sex. (D) Spearman's correlations between baseline inflammatory proteins and fold changes in PBMC-derived S. aureus-induced IL-1 $\beta$, IL-6, and TNF- $\alpha$ responses and $M$. tuberculosis-induced IFN- $\gamma$ responses separated by sex. Significant, positive correlations $(\rho>0)$ are depicted in red, significant negative correlations $(\rho<0)$ in blue, and nonsignificant correlations in white. Only proteins with a significant correlation with at least one of the ex vivo cytokine responses are depicted in this figure. 
Also, our validation cohorts were too small to be analyzed when stratified by sex. Finally, BCG-Denmark was used in the third validation cohort, while BCG-Bulgaria was used in our study and the other 2 validation cohorts. Considering the differences in the immune response induced by different BCG strains $(55,56)$, this might explain some of the discrepancies between the cohort studies.

The findings from this study confirm the immunomodulatory properties of BCG vaccination, but also demonstrate a clear effect of inflammation on (non)specific immunogenicity of BCG vaccination. Our findings are likely to explain at least some of the multiple examples from the literature in which BCG improved or protected against inflammatory, allergic, or autoimmune diseases. More studies are needed to increase our understanding of the interaction between inflammation, epigenetic reprogramming, and cellular metabolism of innate immune cells during induction of trained immunity, as well as possible sex specificity of these effects. Multiple tuberculosis vaccine candidates have now entered clinical trials to potentially replace BCG in the future. These vaccines should also be tested for their ability to induce trained immunity and affect systemic inflammation, as well as for potential sex differences. A better understanding of these effects may help optimize vaccine efficacy and explore novel applications of BCG vaccination.

\section{Methods}

Study design and patient cohorts. To study the immunological effects of BCG vaccination, 307 healthy (male and female) adult volunteers of Western European ancestry were included in the 300BCG cohort between April 2017 and June 2018 in the Radboud University Medical Center. Healthy volunteers were recruited using local advertisements and flyers in Nijmegen, and were compensated for participation. After written informed consent was obtained, blood was collected, followed by administration of a standard dose of $0.1 \mathrm{~mL}$ BCG (BCG-Bulgaria, InterVax) intradermally in the left upper arm by a medical doctor. Vaccination of study participants was organized in batches of 6-16 subjects per day. Two weeks and 3 months after BCG vaccination, additional blood samples were collected. Exclusion criteria were use of systemic medication other than oral contraceptives or acetaminophen, use of antibiotics 3 months before inclusion, previous BCG vaccination, history of tuberculosis, any febrile illness 4 weeks before participation, any vaccination 3 months before participation, and a medical history of immunodeficiency.

A healthy cohort of 493 individuals of Western European descent was used as an independent validation cohort (500FG cohort, see www.humanfunctionalgenomics.org). The participants were recruited between August 2013 and December 2014 at the Radboud University Medical Center (57). In addition, 3 BCG studies conducted at the Radboud University Medical Center, all in BCG-naive participants, were used as independent validation cohorts. In validation cohort 1 , 15 subjects (67\% male, age $18-50$ years) received a standard dose of BCG vaccination (BCG-Bulgaria, InterVax) between January 2017 and April 2017 (4). Blood was drawn before, 2 weeks, and 3 months after vaccination. In validation cohort 2, 9 healthy volunteers (33\% male, age 18-35 years) received a standard dose of BCG vaccination (BCGBulgaria, InterVax) in August 2016 (5). Blood was drawn at baseline and 5 weeks after vaccination. In validation cohort 3,15 male volunteers (age 19-37 years) received a standard dose of BCG vaccination
(BCG-Denmark, SSI) between February 2015 and November 2015 (6). Blood was drawn at baseline and 4 weeks after vaccination.

PBMC isolation and stimulation. PBMCs were isolated from EDTA whole blood with Ficoll-Paque (GE Healthcare) density gradient separation. PBMCs were washed twice with phosphate-buffered saline (PBS) and counted with a Sysmex hematology analyzer (XN-450). Complete blood counts were performed on EDTA whole blood and PBMC fractions after Ficoll isolation on a Sysmex XN-450 hematology analyzer. Cells were suspended in Dutch-modified Roswell Park Memorial Institute (RPMI) 1640 medium (Invitrogen), supplemented with $50 \mu \mathrm{g} / \mathrm{mL}$ gentamycin, $2 \mathrm{mM}$ Glutamax (GIBCO), and $1 \mathrm{mM}$ pyruvate (GIBCO). PBMCs $\left(5 \times 10^{5}\right)$ were cultured in a final volume of $200 \mu \mathrm{L} /$ well in round-bottom 96-well plates (Greiner) and stimulated with RPMI 1640 (medium control), heat-killed M. tuberculosis HR37v (5 $\mathrm{g} / \mathrm{mL}$, specific stimulus), or heat-killed $S$. aureus $\left(1 \times 10^{6} \mathrm{CFU} / \mathrm{mL}\right.$, nonspecific stimulus). Supernatants were collected after 24 hours and 7 days of incubation at $37^{\circ} \mathrm{C}$ and stored at $-20^{\circ} \mathrm{C}$ until analysis. Cytokine levels were measured in 24-hour (IL-1 $\beta$, IL-6, and TNF- $\alpha$ ) and 7-day (IFN- $\gamma$ ) supernatants. Supernatant samples from one participant from different time points were measured on the same plate to ensure that variation between plates would not affect the calculated fold changes.

Protein and hormone measurements. Circulating plasma inflammatory markers were measured before, 2 weeks, and 3 months after BCG vaccination using the commercially available Olink Proteomics AB Inflammation Panel (92 inflammatory proteins), using a Proceek Multiplex proximity extension assay (58). In this assay, proteins are recognized by pairs of antibodies coupled to cDNA strands, which bind when they are in close proximity and extend by a polymerase reaction. A pooled plasma sample and an interplate control were included on each plate in triplicate to correct for batch differences. Plasma samples from one participant from different time points were measured on the same plate to ensure that variation between plates would not affect the calculated fold changes. Detected proteins were normalized according to interplate controls to minimize interassay variation and measured on a $\log _{2}$ scale as normalized protein expression values.

In addition, adiponectin, resistin, and leptin were measured in EDTA plasma at baseline using the R\&D Systems DuoSet ELISA kits following the manufacturer's protocol. Testosterone was also measured in plasma at baseline by LC-MS/MS after protein precipitation and solid-phase extraction as described previously (57). Also, IgG class antibodies against CMV were measured in EDTA plasma using the Genway Biotech ELISA according to the manufacturer's protocol.

Statistics. All computational analyses were performed in R 3.3.3. Proteins were excluded from the analysis when the target protein was detected in less than $75 \%$ of the samples. Protein concentrations under the detection threshold were replaced with the protein's lower limit of detection. Protein circulation concentrations were then correlated with blood counts using Spearman's rank-order correlation. Protein concentrations were compared between baseline and 2 weeks as well as 3 months after BCG vaccination using Wilcoxon's matched-pairs signedrank test. An FDR of less than 0.05 based on the Benjamini-Hochberg procedure was considered significant. Subsequently, blood counts were compared between baseline and 2 weeks as well as 3 months after BCG vaccination using Wilcoxon's matched-pairs signed-rank test. Baseline testosterone, adiponectin, resistin, and leptin levels were correlated with fold changes in circulating inflammatory markers using Spearman's rank-order correlation. Finally, raw cytokine values from the 
PBMC stimulation experiments were first log transformed, and then corrected for batch effects using a linear regression model. Corrected cytokine concentrations were compared between baseline and 2 weeks as well as 3 months after BCG vaccination using Wilcoxon's matchedpairs signed-rank test. Corrected cytokine production was converted to fold changes from baseline. These fold changes were thereafter correlated with baseline inflammatory markers using Spearman's rankorder correlation separated by sex. A 2-sided $P$ value of less than 0.05 was considered statistically significant.

Study approval. The 300BCG (NL58553.091.16) and 500FG (NL42561.091.12) studies were approved by the Arnhem-Nijmegen Medical Ethical Committee. Validation cohorts 1 (NL55825.091.15) and 3 (NL50160.092.24) were also approved by the Arnhem-Nijmegen Medical Ethical Committee, and validation cohort 2 was approved by the Central Committee on Research Involving Human Subjects (CCMO NL56222.091.15). Written informed consent was obtained before any research procedure was initiated. All studies were performed in accordance with the declaration of Helsinki.

\section{Author contributions}

MGN, LCJDB, and VACMK designed the study. VACMK, LCJDB, VPM, SJCFMM, HL, and HD conducted the cohort study and performed the experiments. LCJDB, JW, BC, RJWA, and $\mathrm{MJ}$ conducted the trials used as validation cohorts. VACMK and LCJDB analyzed the data. MGN, RVC, LABJ, and CSB supervised the analysis and interpretation of results. LCJDB and VACMK wrote the manuscript, which was critically reviewed and approved by all authors.

\section{Acknowledgments}

We would like to thank all volunteers from the 300BCG cohort for participation in the study. We thank Trees Jansen for her help with labeling of all study materials, and Robert Sauerwein for accommodating the material we used for validating our results. MGN was supported by a Spinoza grant of the Netherlands Organization for Scientific Research and an European Research Council Advanced Grant (TRAIN-OLD no. 833247). LCJDB was partly funded by a grant to the Research Center for Vitamins and Vaccines (CVIVA) from the Danish National Research Foundation (DNRF108).

Address correspondence to: Mihai G. Netea, Radboudumc, Geert Grooteplein 8, 6525 GA Nijmegen, Netherlands. Phone: 31.24.36618819; Email: mihai.netea@radboudumc.nl.
1. Goodridge HS, et al. Harnessing the beneficial heterologous effects of vaccination. Nat Rev Immunol. 2016;16(6):392-400.

2. de Bree LCJ, et al. Non-specific effects of vaccines: current evidence and potential implications. Semin Immunol. 2018;39:35-43.

3. Kaufmann E, et al. BCG educates hematopoietic stem cells to generate protective innate immunity against tuberculosis. Cell. 2018;172(1-2):176-190.e19.

4. Cirovic B, et al. BCG vaccination in humans elicits trained immunity via the hematopoietic progenitor compartment. Cell Host Microbe. 2020;28(2):322-334.e5.

5 . Walk J, et al. Outcomes of controlled human malaria infection after BCG vaccination. Nat Commun. 2019;10(1):874.

6. Arts RJW, et al. BCG vaccination protects against experimental viral infection in humans through the induction of cytokines associated with trained immunity. Cell Host Microbe. 2018;23(1):89-100.e5.

7. Aaby P, et al. Randomized trial of BCG vaccination at birth to low-birth-weight children: beneficial nonspecific effects in the neonatal period? J Infect Dis. 2011;204(2):245-252.

8. Biering-Sørensen S, et al. Small randomized trial among low-birth-weight children receiving bacillus Calmette-Guérin vaccination at first health center contact. Pediatr Infect Dis J. 2012;31(3):306-308.

9. Biering-Sørensen S, et al. Early BCG-Denmark and neonatal mortality among infants weighing $<2500$ g: a randomized controlled trial. Clin Infect Dis. 2017;65(7):1183-1190.

10. Biering-Sørensen S, Jensen KJ, Monterio I, Ravn $\mathrm{H}$, Aaby P, Benn CS. Rapid protective effects of early BCG on neonatal mortality among low birth weight boys: observations from randomized trials. J Infect Dis. 2018;217(5):759-766.
11. Benn CS, Netea MG, Selin LK, Aaby P. A small jab - a big effect: nonspecific immunomodulation by vaccines. Trends Immunol. 2013;34(9):431-439.

12. Schaltz-Buchholzer F, et al. Early BCG vaccination, hospitalizations, and hospital deaths: analysis of a secondary outcome in 3 randomized trials from Guinea-Bissau. J Infect Dis. 2019;219(4):624-632.

13. Bekkering S, Joosten LA, van der Meer JW, Netea MG, Riksen NP. Trained innate immunity and atherosclerosis. Curr Opin Lipidol. 2013;24(6):487-492.

14. Bekkering S, Quintin J, Joosten LA, van der Meer JW, Netea MG, Riksen NP. Oxidized low-density lipoprotein induces long-term proinflammatory cytokine production and foam cell formation via epigenetic reprogramming of monocytes. Arterioscler Thromb Vasc Biol. 2014;34(8):1731-1738.

15. Christ A, Bekkering S, Latz E, Riksen NP. Long-term activation of the innate immune system in atherosclerosis. Semin Immunol. 2016;28(4):384-393.

16. Bekkering S, et al. Metabolic induction of trained immunity through the mevalonate pathway. Cell. 2018;172(1-2):135-146.e9.

17. Arts RJW, Joosten LAB, Netea MG. The potential role of trained immunity in autoimmune and autoinflammatory disorders. Front Immunol. 2018;9:298.

18. Aaby P, et al. Early BCG vaccination and reduction in atopy in Guinea-Bissau. Clin Exp Allergy. 2000;30(5):644-650.

19. Marks GB, et al. The effect of neonatal BCG vaccination on atopy and asthma at age 7 to 14 years: an historical cohort study in a community with a very low prevalence of tuberculosis infection and a high prevalence of atopic disease. J Allergy Clin Immunol. 2003;111(3):541-549.

20. Kowalewicz-Kulbat M, Locht C. BCG and protection against inflammatory and auto-immune diseases. Expert Rev Vaccines. 2017;16(7):1-10.
21. Steenhuis TJ, et al. Bacille-Calmette-Guerin vaccination and the development of allergic disease in children: a randomized, prospective, single-blind study. Clin Exp Allergy. 2008;38(1):79-85.

22. Thøstesen LM, et al. Neonatal BCG vaccination and atopic dermatitis before 13 months of age: A randomized clinical trial. Allergy. 2018;73(2):498-504.

23. Mangtani $P$, et al. Protection by BCG vaccine against tuberculosis: a systematic review of randomized controlled trials. Clin Infect Dis. 2014;58(4):470-480.

24. von Reyn CF. BCG, latitude, and environmental mycobacteria. Clin Infect Dis. 2014;59(4):607-608.

25. Stensballe LG, et al. BCG vaccination at birth and rate of hospitalization for infection until 15 months of age in Danish children: a randomized clinical multicenter trial. J Pediatric Infect Dis Soc. 2019;8(3):213-220.

26. Blok BA, et al. Interacting, nonspecific, immunological effects of bacille Calmette-Guérin and tetanus-diphtheria-pertussis inactivated polio vaccinations: an explorative, randomized trial. Clin Infect Dis. 2020;70(3):455-463.

27. Mulder WJM, Ochando J, Joosten LAB, Fayad ZA, Netea MG. Therapeutic targeting of trained immunity. Nat Rev Drug Discov. 2019;18(7):553-566.

28. Fourati S, et al. Pre-vaccination inflammation and B-cell signalling predict age-related hyporesponse to hepatitis B vaccination. Nat Commun. 2016;7:10369.

29. Muyanja E, et al. Immune activation alters cellular and humoral responses to yellow fever 17D vaccine. JClin Invest. 2014;124(7):3147-3158.

30. Herr HW, Morales A. History of bacillus Calmette-Guerin and bladder cancer: an immunotherapy success story. JUrol. 2008;179(1):53-56.

31. Bekkering $\mathrm{S}$, et al. Innate immune cell activation and epigenetic remodeling in symptomatic and 
asymptomatic atherosclerosis in humans in vivo. Atherosclerosis. 2016;254:228-236.

32. Ovchinnikova OA, et al. Mycobacterium bovis BCG killed by extended freeze-drying induces an immunoregulatory profile and protects against atherosclerosis. J Intern Med. 2014;275(1):49-58.

33. van Dam AD, et al. BCG lowers plasma cholesterol levels and delays atherosclerotic lesion progression in mice. Atherosclerosis. 2016;251:6-14.

34. Ristori G, et al. Use of bacille CalmetteGuèrin (BCG) in multiple sclerosis. Neurology. 1999;53(7):1588-1589.

35. Paolillo A, et al. The effect of bacille CalmetteGuérin on the evolution of new enhancing lesions to hypointense $\mathrm{T} 1$ lesions in relapsing remitting MS. J Neurol. 2003;250(2):247-248.

36. Ristori G, et al. Effects of bacille CalmetteGuerin after the first demyelinating event in the CNS. Neurology. 2014;82(1):41-48.

37. Kühtreiber WM, et al. Long-term reduction in hyperglycemia in advanced type 1 diabetes: the value of induced aerobic glycolysis with BCG vaccinations. NPJ Vaccines. 2018;3:23.

38. Stienstra R, Netea MG. Firing up glycolysis: BCG vaccination effects on type 1 diabetes mellitus. Trends Endocrinol Metab. 2018;29(12):813-814.

39. Harada M, Kishimoto Y, Makino S. Prevention of overt diabetes and insulitis in NOD mice by a single BCG vaccination. Diabetes Res Clin Pract. 1990;8(2):85-89.

40. Yagi H, Matsumoto M, Kishimoto Y, Makino S, Harada M. Possible mechanism of the preventive effect of BCG against diabetes mellitus in NOD mouse. II. Suppression of pathogenesis by macrophage transfer from BCG-vaccinated mice. Cell Immunol. 1991;138(1):142-149.
41. Thøstesen LM, et al. Neonatal BCG has no effect on allergic sensitization and suspected food allergy until 13 months. Pediatr Allergy Immunol. 2017;28(6):588-596.

42. Gofrit ON, Bercovier H, Klein BY, Cohen IR, Ben-Hur T, Greenblatt CL. Can immunization with bacillus Calmette-Guérin (BCG) protect against Alzheimer's disease? Med Hypotheses. 2019;123:95-97.

43. Zuo Z, et al. Immunization with bacillus Calmette-Guérin (BCG) alleviates neuroinflammation and cognitive deficits in APP/PS1 mice via the recruitment of inflammationresolving monocytes to the brain. Neurobiol Dis. 2017;101:27-39.

44. Quintin J, et al. Candida albicans infection affords protection against reinfection via functional reprogramming of monocytes. Cell Host Microbe. 2012;12(2):223-232.

45. Marchant A, et al. Newborns develop a Th1-type immune response to Mycobacterium bovis bacillus Calmette-Guérin vaccination. J Immunol. 1999;163(4):2249-2255.

46. Stensballe LG, et al. Acute lower respiratory tract infections and respiratory syncytial virus in infants in Guinea-Bissau: a beneficial effect of BCG vaccination for girls community based case-control study. Vaccine. 2005;23(10):1251-1257.

47. Roth A, et al. Tuberculin reaction, BCG scar, and lower female mortality. Epidemiology. 2006;17(5):562-568.

48. Aaby P, Jensen H, Walraven G. Age-specific changes in the female-male mortality ratio related to the pattern of vaccinations: an observational study from rural Gambia. Vaccine. 2006;24(22):4701-4708.
49. Aaby P, et al. Vaccinia scars associated with better survival for adults. An observational study from Guinea-Bissau. Vaccine. 2006;24(29-30):5718-5725.

50. Garly ML, et al. Hepatitis B vaccination associated with higher female than male mortality in Guinea-bissau: an observational study. Pediatr Infect Dis J. 2004;23(12):1086-1092.

51. Aaby P, Ravn H, Fisker AB, Rodrigues A, Benn CS. Is diphtheria-tetanus-pertussis (DTP) associated with increased female mortality? A meta-analysis testing the hypotheses of sexdifferential non-specific effects of DTP vaccine. Trans R Soc Trop Med Hyg. 2016;110(10):570-581.

52. de Bree LCJ, et al. The impact of sex hormones on BCG-induced trained immunity. J Leukoc Biol. 2018;104(3):573-578.

53. Kurtoğlu S, Baştuğ O. Mini puberty and its interpretation. Turk Pediatri Ars. 2014;49(3):186-191.

54. Joosten SA, et al. Mycobacterial growth inhibition is associated with trained innate immunity. J Clin Invest. 2018;128(5):1837-1851.

55. Ritz N, et al. The influence of bacille Calmette-Guerin vaccine strain on the immune response against tuberculosis: a randomized trial. Am J Respir Crit Care Med. 2012;185(2):213-222.

56. Angelidou A, et al. Licensed bacille CalmetteGuérin (BCG) formulations differ markedly in bacterial viability, RNA content and innate immune activation. Vaccine. 2020;38(9):2229-2240.

57. Ter Horst R, et al. Host and environmental factors influencing individual human cytokine responses. Cell. 2016;167(4):1111-1124.e13.

58. Assarsson E, et al. Homogenous 96-plex PEA immunoassay exhibiting high sensitivity, specificity, and excellent scalability. PLoS One. 2014;9(4):e95192. 